\title{
Identifying validated nursing quality indicators for the intensive care unit: an
}

\section{integrative review}

\author{
*Efi Evangelou RN; PhD, BSc, MSc, Research Associate, Department of Nursing, School of Health Sciences, Cyprus \\ University of Technology, Limassol, Cyprus \\ Ekaterini Lambrinou RN; BSc, MSc, PhD, Associate Professor, Department of Nursing, School of Health Sciences, Cyprus \\ University of Technology, Limassol, Cyprus
}

Christiana Kouta RN; BSc, MSc, PhD, Associate Professor, Department of Nursing, School of Health Sciences, Cyprus University of Technology, Limassol, Cyprus

Nicos Middleton BSc, MSc, PhD, Associate Professor, Department of Nursing, School of Health Sciences, Cyprus University of Technology, Limassol, Cyprus

Key words: AIRE instrument intensive care units $*$ nurse staffing nursing care patient outcomes quality indicators

*Email: ekaterini.lambrinou@cut.ac.cy

\section{SUMMARY}

- Background: Quality indicators (QIs) play an important role in evaluating quality improvement initiatives. A generally accepted set of QIs specific to the nursing care in the intensive care unit (ICU) is not available.

- Aim: To identify Qls associated with nursing care for adult ICU in the literature. The methodological quality of QIs was assessed and associated variables of quality and quantity of nursing care were also identified.

- Methods: We employed an integrative literature review. A focused search of electronic databases was applied. Inclusion and exclusion criteria were used for the selection of relevant articles. Quality assessment of the included studies was based on the guidance document of domains and elements suggested by the Agency of Health Care Research and Quality. Qls' methodological quality was assessed using the Appraisal of Indicators through Research and Evaluation (AIRE) instrument. Results: The review identified 13 studies and 45 QIs associated with nursing care in the ICU. The set of QIs assessed in each study, the type of nurse staffing measure as well as morbidity and mortality rates varied considerably. Findings suggest that quality and quantity of nursing care are strongly associated with higher rates of adverse events, mortality, infections and complications in adult ICUs. Methodological quality of the QIs also differed considerably. Higher AIRE scores, indicating higher scientific evidence of methodological quality, can be used to select evidence-based and valid QIs.

- Conclusions: A number of QIs quantifying nursing care in the ICU have been identified. These QIs could be combined to form a tool which would allow to the quantification and assessment of the quality of ICU nursing care provided in a regular basis.

\section{INTRODUCTION}

Care on intensive care units (ICUs) represents a major portion of health care costs and thus consumes a large part of a hospital's financial' resources (Robert et al., 2000). In the ICU, adverse events and human error constitute substantial morbidity and mortality risks to critically ill patients; especially as a result of understaffing (De Vos et al., 2007).

Quality indicators (QIs) play an important role in quality improvement initiatives as long as they are based on evidence (Brook et al., 1996). Nursing care is considered a critical factor of patient care and thus, the assessment of Qls which specifically reflect nurses' contribution is vital (Montalvo, 2007). The identification of QIs that quantify the quality of nursing care would enable the development of sets that could be easily adopted in practice in the context of auditing performance and monitoring evidence-based practice in routine care (Mainz, 2003).

In essence, QIs are screening tools, which identify potential suboptimal clinical care and reveal specific problematic areas that need further investigation (De Vos et al., 2007). Qls may be classified according to three dimensions, which correspond to structure, process and outcome components of quality (Donabedian, 1992). Outcome indicators, in particular, reflect the effect of quality of patients' health care (Mainz, 2003). Thus, potential QIs of nursing care quality may be identified by the extent to which they indicate nurse sensitive outcomes (NSOs). NSOs refer to aspects of patients' experience, behavior or health clinical status, which are completely or partially determined by the quantity and quality of nursing care received. These may include outcomes, which are influenced by several factors, as long as there is evidence to suggest that they are also associated with nursing care (Montalvo, 2007). Nursing Qls are specific to nursing and may thus differ from medical indicators of care quality. They have been defined as those which are based on "nurses' scope and domain of practice, and for which there is empirical evidence linking nursing inputs and interventions to the outcome" (Doran and Pringle 2003, vii). It is important for nursing Qls to form a valid and reliable means of assess nursing care quality (Heslop and Lu 2014). The QIs in the ICU should be relevant to the problem, understandable, measurable, behaviourable (and thus, amenable to change), achievable and feasible, according to the RUMBA rule (Braun et al., 2010).

Most of the published studies examining the relationship between nurse staffing and patient outcomes have been performed in 
general wards or at the entire hospital level (Griffiths et al., 2008, Needleman et al., 2002, Van den Heede et al., 2007). National forums and agencies have proposed a number of QIs. Nevertheless, specific QIs for the ICU have not been suggested (AHRQ, 2006, ANA, 2000, JCAHO, 2007, NQF, 2004). Furthermore, forums and societies specific to the ICU propose QIs, but none is specifically and exclusively referred to as nursing-related QIs (Delgado et al., 2005, Ray et al., 2009). Currently, there is not a complete set of Qls specific to the nursing care in the ICU available (McGahan et al., 2012). In an effort to fill the gap, this study reviews the literature and pools potential QIs associated with nursing care in the ICU. These QIs may be used for the development of nursing QI sets (NQI) specific to the ICU, currently missing from the scientific literature. Identifying a select set of evidence-based and valid NQI offers the potential to assess nursing care delivered in the ICU.

\section{AIM}

To review the literature in order to identify potential Qis, specifically patient-centered clinical NSOs, that may be measured in the ICU and have been found to be associated with variables reflecting the quantity and/or quality of nursing care (i.e. nursing and setting structure variables). The secondary aim was the assessment of the methodological quality of the QIs identified.

\section{METHODS}

\section{Design}

This is an integrative review of published literature based on a modified framework for review research (Whittemore and Knafl 2005). The process for data extraction and synthesis was based on a detailed pre-specified protocol, as presented in the sections to follow.

\section{Search methods}

The process of searching the literature was performed based on the Center of Review and Dissemination guidance (CRD, 2009). A focused search of electronic databases Ovid Medline, PubMed, Cumulative Index of Nursing and Allied Health Literature (CINAHL) and Cochrane library was applied to identify the relevant literature. The time frame of interest was 2000-2016.

The search was undertaken by using the following keywords and medical subject heading (Mesh) terms: "intensive care unit(s)" [Mesh] OR "ICU", "quality indicators- health care" [Mesh] OR "clinical indicators", and nurs* (nurses, nursing, nurse staffing, personnel staffing, critical care nursing) [Mesh]. The search terms were used in all possible combinations using Boolean operators. The "similar articles" tool of PubMed was also used. Furthermore, the reference lists from included studies were also reviewed in order to identify additional studies that may have not been identified in the original search. Authors did not attempt any hand searching of journals, conferences' or abstracts' proceedings.

Variables of interest referred to the education and certification level, years of experience, hours of nursing care per patient day, workload intensity and overtime. Nurse staffing measures such as staff mix, skill mix, staff ratio, and nurse to patient (N/P) ratio were also considered of interest. Nurse staffing measures are already recognized as structure QIs. In each one of the selected studies these measures were examined for possible association with patients' outcomes. Any ICU patient-related clinical outcome was extracted and considered as a potential QI.

Specific inclusion criteria were applied. Only published research articles were selected. Studies were included if they had examined associations between nursing and setting related structure variables with critically ill adult patients' outcomes. Studies that evaluated QIs already recognized as NSOs were also included. Secondary data analysis surveys were also included. Only quantitative studies were identified and included. For candidate QIs in any of the identified studies, the numerator and denominator of Qls should be given or at least it should be easily understood from the description. The relativeness to any type of ICU was set as an additional selection criterion.

Articles published in language other than English, unpublished studies, abstracts, editorials, expert opinion papers, secondary sources, such as reviews or systematic literature reviews, conceptual sources, anecdotal and opinion sources were excluded. Studies that assessed the association of Qls with nurse staffing outside the hospital, after discharge from the ICU, at the ward or during transfer from the ICU, were excluded. Studies that presented results at a hospital level were also excluded. Other structure components of quality and organizational characteristics such as daily rounds and team work were not considered.

\section{Quality appraisal}

The methodological quality of the included studies was independently assessed by two of the authors and was based on the domains and elements suggested by the Agency of Health Care Research and Quality. The assessment considered: study question, population, comparability of subjects, exposure or intervention, outcome, statistical analysis, results, discussion, funding or sponsorship (West et al., 2002).

Additionally, the methodological quality of the included Qls was assessed using the Appraisal of Indicators through Research and Evaluation (AIRE) instrument. The AIRE instrument is a new tool, which was designed and validated in the Netherlands (De Roo et al., 2013). It was previously used in peer-reviewed studies aiming to develop a set of QIs for palliative care (De Roo et al., 2013), for midwifery care (De Bruin-Kooistra et al., 2012), of musculoskeletal injury management (Strudwick et al., 2015) and care of osteoarthritis (Petrosyan et al., 2017). AIRE addresses the face and construct validity, accuracy, risk of bias, ability to achieve real quality improvement, and application of Qls (Strudwick et al., 2015).

In this review, the AIRE instrument was used to assess whether the objective and the organizational background of the identified QIs are well defined and the extent to which they built on evidence (Smeulers et al., 2015). AIRE contains four domains that examine: 1. Purpose, relevance and organizational context of the QIs, 2. Stakeholders' involvement for the development of the QIs, 3. Scientific evidence and 4. Additional evidence, formulation, usage. Additionally, there are in total twenty items (e.g. "systematic methods were used for search for scientific evidence") across these four domains. Each of the four authors independently scored on a 4 point Likert scale ( $1=$ strongly disagree and $4=$ agree) for the items given (De Roo et al., 2013). The maximum score for an item is 16 and the minimum is 4 , when four authors are asked to rate. In the present study AIRE was applied for the set of the QIs identified in each study, rather than for each QI separately (Smeulers et al., 2015). In the absence of guidance regarding definition of high, medium and low scores for the items, the authors considered as high: 16-14, medium: 13-9 and low: 4-8. Higher scores are indicating valid and widely used QIs. A score of $50 \%$ and higher in all four domains indicates higher methodological quality (Strudwick et al., 2015).

\section{Data extraction}

A structured data extraction form was used to collect information from the studies, which are summarized in Table 1. The data extraction form included: 1. Authors/ publication date, Country and Data collection period, 2. Research aim(s), 3. Methods, and 4. Quality indicators and formula or definition 5 . Nurse staffing variables and 
definition and 6. Results (main). The initial selection included the screening of titles and abstracts against the inclusion criteria. The second stage is referred to the screening of the full papers in order to identify articles that fulfill the inclusion criteria. Two of the authors extracted information and independently reviewed eligibility criteria of the articles obtained. Any disagreements were resolved through consensus, and when necessary, with the involvement of the third author. The process ended up with full consensus after detailed examination of full text articles and consideration of predetermined inclusion criteria. In the case of incomplete information the article was excluded. A flow diagram of the search strategy is depicted in Figure 1.

\section{Data synthesis}

Similar data were categorized and organized together so as to enable comparison and interpretation (Table 1). Moreover, Qls were categorized as primary (e.g. mortality, cost) and secondary (e.g. infections, complications) (Amavardi et al., 2000, Pronovost et al., 1999) and further classified based on a previously described framework (Doran \& Pringle, 2011, Holzemer 1994). The subcategories included: setting- related outcome variables (QIs) and patient safety related outcome (Heslop \& Lu, 2014), as shown in Table 2.

\section{RESULTS}

\section{Search outcomes}

The search yielded 83 article titles of which only five articles were selected for further analysis based on the methodological assessment. Full text was obtained for all five articles and eight additional studies were identified, either from the reference lists, or classified by the database as related articles to those already obtained. A total of 13 studies were included in this review (Figure 1). The studies refer to 45 Qls in total (Table 1 and 2).

\section{Overview of the included studies}

There is a growing interest worldwide regarding patients' outcomes that are affected by the nursing care provided. The included studies originated from Asia (Cho et al., 2008), Switzerland (Bracco et al., 2001), Scotland (Tarnow-Mordi et al., 2000), Austria (Metnitz et al., 2008), one was a multi-centered worldwide study (Valentin et al., 2006) and the remaining eight studies originated from the USA.

Across the selected studies, the respective ICUs differed in type and included surgical, medical and mixed ICUs. The number of participating ICUs ranged from eight (Alonso- Echanove et al., 2003) to as many as 205 (Valentin et al., 2006). The number of involved hospitals and their capacity also differed, while, as shown in Table 1 , a number of studies did not report the type or number of included hospitals (Kendall-Gallagher and Blegen, 2009, Metnitz et al., 2008, Valentin et al., 2006).

\section{Quality appraisal}

All included studies used an observational design. Six studies used prospective data (Alonso- Echanove et al., 2003, Bracco et al., 2001, Garcia and Fugulin, 2012, Metnitz et al., 2008, Robert et al., 2000, Valentin et al., 2006) and seven studies collected retrospective data (Amavardi et al., 2000, Cho et al., 2008, Dang et al., 2002, KendallGallagher and Blegen, 2009, Stone et al., 2007, Tarnow-Mordi et al., 2000, Whitman et al., 2002). Data collection methods included: 1. Questionnaires regarding ICU and/or hospital characteristics which were completed by the medical director of the unit (Amavardi et al., 2000, Dang et al., 2002, Metnitz et al., 2008) or the head nurse of the unit (Tarnow-Mordi et al., 2000) of ICUs, 2. Forms specifically designed by the research teams which were completed by MDs and nurses (Alonso- Echanove et al., 2003; Bracco et al., 2001; Robert et al., 2000; Valentin et al., 2006) or infection control practitioners (Alonso-Echanove et al., 2003). One study (Whitman et al., 2002) used a combination of qualitative and quantitative data collection through interviews and special forms (Table 1).

All of the studies included a focused aim or a research question (Table 1). Only three studies used Qls already recognized as nursing specific (Bracco et al., 2001; Valentin et al., 2006; Whitman et al., 2002). The remaining ten studies clearly aimed to explore the association between structural variables and patient outcome(s).

Sample sizes of the included studies ranged from 28 (Robert et al., 2000) to 83259 (Metnitz et al., 2008) patients. Two studies do not clearly report specific inclusion and exclusion criteria for the sample (Garcia and Fugulin, 2012, Kendall-Gallagher and Blegen, 2009). The majority of the sample employed multivariable analyses (Alonso -Echanove et al., 2003; Amavardi et al., 2000; Bracco et al., 2001; Kendall-Gallagher \& Blegen, 2009; Metnitz et al., 2008; Stone et al., 2007; Tarnow-Mordi et al., 2000).

Five studies report funding (Cho et al. 2008, Kendall-Gallagher and Blegen, 2009, Stone et al., 2007, Valentin et al., 2006, Whitman et al., 2002), whereas ethical approvals were sought and granted in six of the selected studies (Amavardi et al., 2000; Bracco et al., 2001; Garcia \& Fugulin, 2012; Kendall-Gallagher \& Blegen, 2009; Metnitz et al., 2009; Whitman et al., 2002).

Table 3 shows the scores of the Qls based on AIRE (De Roo et al., 2013). The highest scores were obtained for items 18 (97\%) and $10(96 \%)$ indicating that the Qls had been tested in daily practice and were evidence-based, respectively. Most of the sets achieved the highest scores (89\%) for category III "Scientific evidence" and the lowest $(51 \%)$ for the category II "Stakeholder involvement" (Smeulers et al., 2015). Only Dang et al. (2002) and Amavardi et al. (2000) reported the development process and recruited a panel to identify ICD-9-CM codes for the QIs. The remaining eleven studies applied QIs that have been widely used in previous studies. All of the included studies provide the definitions or the formulas for calculation of QIs. None of the included studies provided full description of terminology, rationality or justification, source of the data and type of the parameter.

\section{Quality indicators identified}

Findings of the included studies regarding rate measures of Qls and main associations identified in each study are presented in Table 1. The QIs identified were grouped and classified in subgroups (Table 2). The domain that appears to be mostly covered is safety (Doran \& Pringle, 2011). Negative performance Qls were most common, such as adverse events, infections and complications (Mitchell, 2008). Most commonly used QIs are mortality and blood stream infections (BSIs).

There are Qls with a variety of names that examine the same numerators and denominators such as decubitus ulcers (Stone et al., 2007) and skin break down (Kendall-Gallagher and Blegen, 2009). However, in the case that different numerators and denominators are used for seemingly identical Qls, these were regarded as different outcomes. Likewise, there are similar Qls that may be considered part of a more general QI, such as device related catheter-associated urinary tract infection (CAUTI) and urinary tract infection (UTI). Identified definitions are provided in Table 1.

Valentin et al. (2006) and Bracco et al. (2001) used sentinel Qls (undesirable events that trigger further investigation) whereas the remaining studies applied rate-based QIs. In some studies, QIs were restricted to specific patient groups of the ICU. Robert et al. (2000) and Alonso-Echanove et al. (2003) focused on patients with central venous catheter (CVC). Amavardi et al. (2000) examined patients with esophageal resection and Dang et al. (2002) was interested in patients who underwent abdominal aortic surgery. Stone et al. (2007) focused on elderly patients. 


\begin{tabular}{|c|c|c|c|c|c|}
\hline $\begin{array}{l}\text { Authors, } \\
\text { publication date, } \\
\text { country }\end{array}$ & Research aim(s) & $\begin{array}{l}\text { Methods: study } \\
\text { design, population, } \\
\text { tools used study } \\
\text { duration }\end{array}$ & $\begin{array}{l}\text { Qls and formula or } \\
\text { definition identified }\end{array}$ & $\begin{array}{l}\text { Nurse staffing variables } \\
\text { and definition identified }\end{array}$ & Results \\
\hline $\begin{array}{l}\text { Kendall-Gallagher \& } \\
\text { Blegen, 2009, USA }\end{array}$ & $\begin{array}{l}\text { To explore: } \\
\text { the relationship } \\
\text { between the } \\
\text { proportion of } \\
\text { certified staff } \\
\text { nurses and the } \\
\text { risk of harm of } \\
\text { patients } \\
\text { the organizational } \\
\text { and nursing } \\
\text { characteristics } \\
\text { associated with } \\
\text { rates of adverse } \\
\text { events }\end{array}$ & 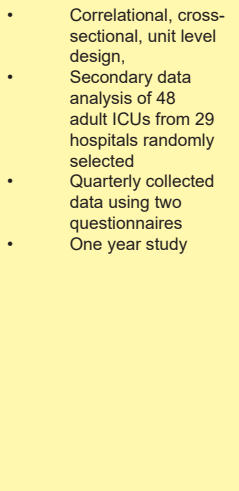 & $\begin{array}{l}\text { Falls: annual rate per } \\
\text { 1000 patient days } \\
\text { Medication } \\
\text { administration errors } \\
\text { (MAEs): annual rate per } \\
\text { 1000 patient days } \\
\text { Skin break down: } \\
\text { annual rate per } 1000 \\
\text { patient days } \\
\text { Urinary tract infection } \\
\text { (UTI): annual rate per } \\
\text { 1000 patient days } \\
\text { Central catheter line } \\
\text { infection (CCLI): annual } \\
\text { rate per 1000 patient } \\
\text { days } \\
\text { Bloodstream infection } \\
\text { (BSI): annual rate per } \\
\text { 1000 patient days }\end{array}$ & $\begin{array}{l}\text { Registered nurses } \\
\text { (RNs) education level: } \\
\text { percentage of RNs with } \\
\text { bachelor of science } \\
\text { or higher education in } \\
\text { nursing } \\
\text { RN years of experience: } \\
\text { mean years of } \\
\text { experience of RNs } \\
\text { Total hours of nursing } \\
\text { care per day: mean } \\
\text { total working hours of all } \\
\text { nursing staff per day } \\
\text { RN skill mix: percentage } \\
\text { of nursing staff who are } \\
\text { registered nurses } \\
\text { RN work group } \\
\text { competence: percentage } \\
\text { of certified staff RNs }\end{array}$ & 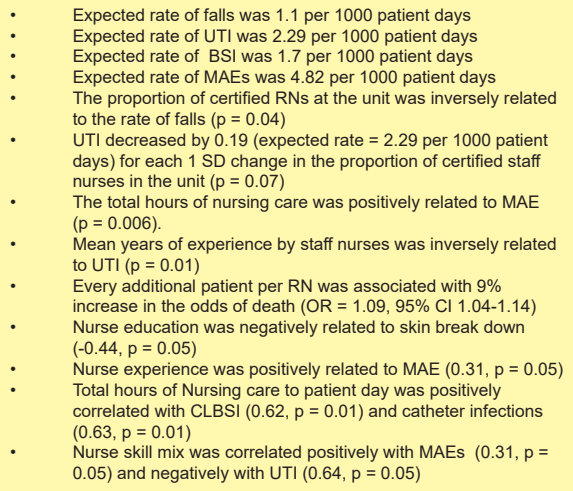 \\
\hline $\begin{array}{l}\text { Cho et al., } 2008 \text {, } \\
\text { Korea }\end{array}$ & $\begin{array}{l}\text { To examine the } \\
\text { relationship between } \\
\text { nurse staffing and patient } \\
\text { mortality in ICUs }\end{array}$ & $\begin{array}{l}\text { Correlational study } \\
\text { collecting data } \\
\text { from administrative } \\
\text { databases } \\
27,372 \text { ICU patients } \\
\text { discharged from } \\
42 \text { tertiary and } 194 \\
\text { secondary hospitals } \\
\text { (total } 236 \text { hospitals) } \\
\text { aged >15 years } \\
\text { old with } 26 \text { primary } \\
\text { diagnoses } \\
3 \text { data sources were } \\
\text { used: ICU survey } \\
\text { data (hospital and } \\
\text { ICU characteristics); } \\
\text { Medical claims data } \\
\text { (patients clinical } \\
\text { and utilization } \\
\text { information); and } \\
\text { NHI (enrollee } \\
\text { database for death } \\
\text { day) } \\
3 \text { months }\end{array}$ & $\begin{array}{l}\text { Mortality: deaths that } \\
\text { occurred in the hospital } \\
\text { or on the date of } \\
\text { hospital discharge }\end{array}$ & $\begin{array}{l}\text { Nurses' years of work } \\
\text { experience: the years of } \\
\text { RNs' license to the time } \\
\text { yr of data collection } \\
\text { Staffing of RNs: ratio of } \\
\text { average daily census to } \\
\text { the total number of full } \\
\text { time equivalent RNs in } \\
\text { ICUs (ADC/RN ratio) }\end{array}$ & 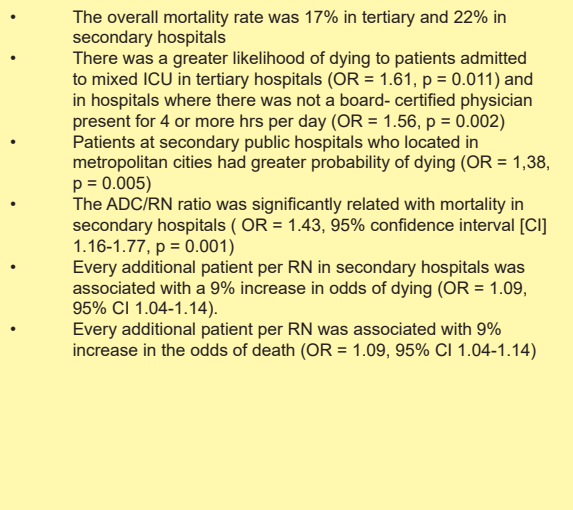 \\
\hline $\begin{array}{l}\text { Robert et al., } 2000, \\
\text { USA }\end{array}$ & $\begin{array}{l}\text { To determine the risk } \\
\text { factors for primary BSI, } \\
\text { including the effect of } \\
\text { RNs' levels }\end{array}$ & $\begin{array}{l}\text { - Nested case control } \\
\text { study } \\
28 \text { patients with } \\
\text { primary BSI (case) } \\
\text { compared with } 99 \\
\text { randomly selected } \\
\text { patients (control) } \\
\text { hospitalized for } \\
\text { more than } 3 \text { days of } \\
\text { a } 20 \text {-bed Surgical } \\
\text { ICU in a } 1000 \text { bed } \\
\text { university- affiliated } \\
\text { inner city teaching } \\
\text { public hospital } \\
\text { A standardized } \\
\text { form was used for } \\
\text { data collection, as } \\
\text { well as the relative } \\
\text { microbiological data } \\
\text { 1-year study period }\end{array}$ & $\begin{array}{l}\text { Primary BSI: BSI were } \\
\text { defined according to the } \\
\text { CDC criteria per } 1000 \\
\text { patient days }\end{array}$ & $\begin{array}{l}\text { Composition of nursing } \\
\text { staff (pool versus regular } \\
\text { nurses): } \\
\text { Regular staff: nurses } \\
\text { permanently assigned } \\
\text { to the unit } \\
\text { Pool staff: nursing staff } \\
\text { that are members of the } \\
\text { hospital pool service or } \\
\text { agency nurses who work } \\
\text { at the hospital } \\
\text { Mean of nurse to patient } \\
\text { ratio (N/P) is expressed } \\
\text { as the maximum number } \\
\text { of nursing hours per } \\
\text { SICU-patient day }\end{array}$ & 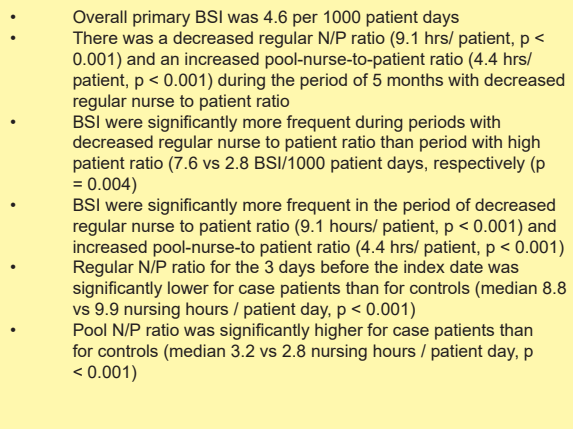 \\
\hline $\begin{array}{l}\text { Dang et al., 2002, } \\
\text { USA }\end{array}$ & $\begin{array}{l}\text { To isolate the effects } \\
\text { of nurse staffing on } \\
\text { patient's outcomes } \\
\text { (medical complications } \\
\text { associated with mortality } \\
\text { and are nurse sensitive) } \\
\text { by examining the } \\
\text { association between } \\
\text { ICU nurse staffing and } \\
\text { the likelihood of medical } \\
\text { complications for patient } \\
\text { undergoing abdominal } \\
\text { aortic surgery }\end{array}$ & $\begin{array}{l}\text { Retrospective study } \\
\text { with secondary } \\
\text { analysis of hospital } \\
\text { discharge data } \\
2606 \text { patients } \\
\text { aged } 30 \text { years or } \\
\text { older with principal } \\
\text { procedure code for } \\
\text { abdominal aortic } \\
\text { surgery, } \\
38 \text { ICUs in one state } \\
\text { Data were obtained } \\
\text { by: Health discharge } \\
\text { data set was } \\
\text { used for patient } \\
\text { information; A } \\
\text { questionnaire was } \\
\text { used for nurse } \\
\text { staffing data; An } \\
\text { instrument of } 32 \\
\text { items was used for } \\
\text { the organizational } \\
\text { characteristics; } \\
\text { and a panel of } \\
\text { experts including } \\
4 \text { physicians who } \\
\text { identified the } \\
\text { ICD-9-CM codes } \\
\text { for the potential } \\
\text { complications }\end{array}$ & $\begin{array}{ll}\text { Complications related } \\
\text { to mortality and are } \\
\text { nurse sensitive: } \\
\text { Cardiac complications: } \\
\text { - acute MI (ICD-9-CM } \\
\text { code: } 410 \text { ); arrest (ICD- } \\
\text { 9-CM code: } 4275 \text { ) } \\
\text { Complications after a } \\
\text { procedure (ICD-9-CM } \\
\text { code: } 9971 \text { ) } \\
\text { Respiratory } \\
\text { complications: } \\
\text { pulmonary insufficiency } \\
\text { after a procedure } \\
\text { (ICD-9-CM codes: } \\
\text { 5184, 5185, 5188); } \\
\text { reintubation (ICD-9-CM } \\
\text { code: } 9604 \text { ); aspiation } \\
\text { ICD-9-CM codes: 507, } \\
\text { 9973; ventilation > 96 } \\
\text { hrs ICD-9-CM code: } \\
\text { 9672 } \\
\text { Others complications: } \\
\text { acute renal failure } \\
\text { ICD-9-CM code: } 584 ; \\
\text { septicemia: ICD-9-CM } \\
\text { code: } 038 ; \text { platelets } \\
\text { transfusion ICD-9-CM } \\
\text { code: } 9905\end{array}$ & $\begin{array}{ll}\text { Intensity of nursing staff: } \\
\text { the average N/P ratio at } \\
\text { day and night } \\
\text { Low, medium and high } \\
\text { intensity nurse staffing } \\
\text { at day and night was } \\
\text { calculated }\end{array}$ & 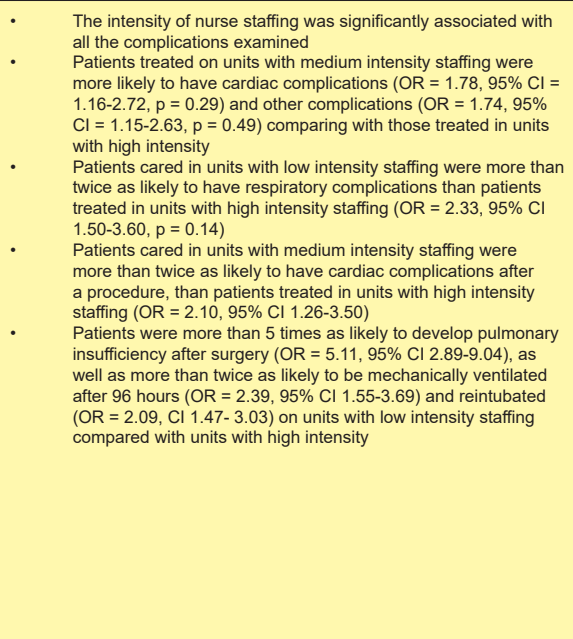 \\
\hline
\end{tabular}




\begin{tabular}{|c|c|c|c|c|c|}
\hline $\begin{array}{l}\text { Authors, } \\
\text { publication date, } \\
\text { country }\end{array}$ & Research aim(s) & $\begin{array}{l}\text { Methods: study } \\
\text { design, population, } \\
\text { tools used study } \\
\text { duration }\end{array}$ & $\begin{array}{l}\text { QIs and formula or } \\
\text { definition identified }\end{array}$ & $\begin{array}{l}\text { Nurse staffing variables } \\
\text { and definition identified }\end{array}$ & Results \\
\hline $\begin{array}{l}\text { Amavardi et al., 2000, } \\
\text { USA }\end{array}$ & $\begin{array}{l}\text { To determine if the } \\
\text { presence of N/P ratio } \\
\text { at night time (NNPR) of } \\
\text { one nurse caring for one } \\
\text { or two patients vs one } \\
\text { nurse caring for three or } \\
\text { more patients in the ICU } \\
\text { is associated with clinical } \\
\text { and economic outcomes } \\
\text { following esophageal } \\
\text { resection }\end{array}$ & $\begin{array}{l}\text { Statewide } \\
\text { observational cohort } \\
\text { study } \\
353 / 366 \text { adult ICU } \\
\text { surgical patients } \\
\text { aged } 18 \text { years and } \\
\text { older in } 32 / 35 \text { acute } \\
\text { care hospitals with } \\
\text { primary procedure } \\
\text { code of esophageal } \\
\text { resection/ } \\
\text { Data were obtained } \\
\text { from: the hospital } \\
\text { discharged } \\
\text { database; } \\
\text { previously validated } \\
\text { questionnaire with } \\
32 \text { organizational } \\
\text { characteristics; } \\
\text { ICD-9-CM codes } \\
\text { including primary } \\
\text { diagnosis, } 14 \\
\text { secondary discharge } \\
\text { diagnoses and } \\
\text { 14 secondary } \\
\text { procedures; a } \\
\text { panel of } 2 \text { ICU } \\
\text { physicians for } \\
\text { selected secondary } \\
\text { outcomes that reflect } \\
\text { post- operative } \\
\text { complications/ } \\
\text { 4-year study }\end{array}$ & $\begin{array}{l}\text { Primary: mortality; } \\
\text { hospital LOS; cost } \\
\text { Secondary: post- } \\
\text { operative infection } \\
\text { (ICD-9-CM code:9985); } \\
\text { aspiration (ICD-9-CM } \\
\text { codes: 507, 9973); } \\
\text { reintubation (ICD-9-CM } \\
\text { code: 9604); pulmonary } \\
\text { insufficiency (ICD-9-CM } \\
\text { codes: } 5184,5185, \\
\text { 5188); pneumonia } \\
\text { (ICD-9-CM codes: } \\
\text { 480-487); septicemia } \\
\text { ICD-9-CM code: } 038 ; \\
\text { cardiac complications } \\
\text { (ICD-9-CM code: } \\
\text { 9971); cardiac arrest } \\
\text { (ICD-9-CM code: } 4257) ; \\
\text { acute MI (ICD-9-CM } \\
\text { code: } 410 \text { ); renal failure } \\
\text { (ICD-9-CM code: } 584 \text { ); } \\
\text { reoperation for bleeding } \\
\text { (ICD-9-CM codes: } \\
\text { 3941, 3949, 3998); } \\
\text { surgical complications } \\
\text { (ICD-9-CM codes: } \\
\text { 9981, 9982, 9983) }\end{array}$ & $\begin{array}{l}\text { - N/P ratio at night shift } \\
\text { (NNPR): } \\
\text { Nurse caring for }>1: 2 \\
\text { means that } 1 \text { nurse is } \\
\text { caring for } 1 \text { or } 2 \text { patients } \\
\text { Nurse caring for }<1: \\
2 \text { means that } 1 \text { nurse } \\
\text { is caring for } 3 \text { or more } \\
\text { patients }\end{array}$ & 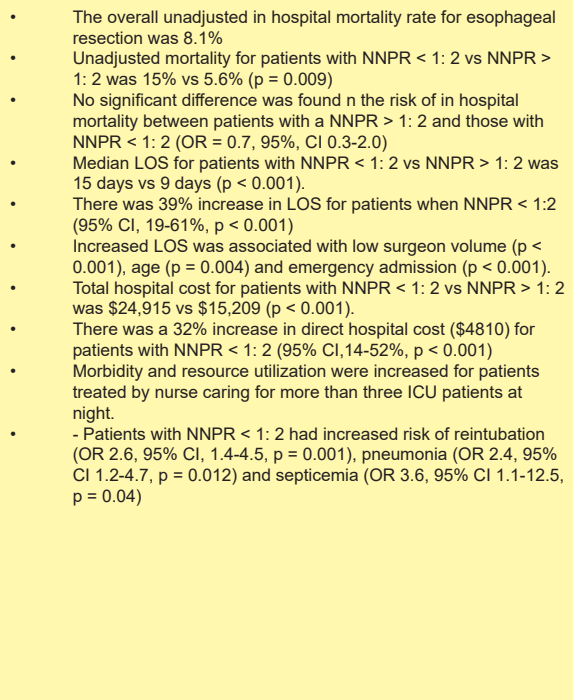 \\
\hline $\begin{array}{l}\text { Alonso-Echanove et } \\
\text { al., 2003, USA }\end{array}$ & $\begin{array}{l}\text { To evaluate the role of } \\
\text { the patient, CVC and } \\
\text { nurse staffing factors } \\
\text { in the risk of CVC- } \\
\text { associated BSI }\end{array}$ & $\begin{array}{l}\text { Prospective, } \\
\text { observational } \\
\text { multicenter, cohort } \\
\text { study conducted } \\
\text { in a non- research } \\
\text { setting } \\
\text { 4535 adults } \\
\text { in } 8 \text { ICUs at } 6 \\
\text { district hospitals } \\
\text { representing } 8593 \\
\text { CVCs and } 56627 \\
\text { catheter days } \\
\text { Data collection was } \\
\text { based on NNIS } \\
\text { system and then } \\
\text { transmitted to CDC } \\
3 \text { data collection } \\
\text { forms were } \\
\text { developed: patient } \\
\text { admission form; } \\
\text { daily log form; CVC } \\
\text { log form }\end{array}$ & $\begin{array}{l}\text { CVC associated with } \\
\text { BSI: according to the } \\
\text { NNIS system definition } \\
\text { per } 1000 \text { CVC days }\end{array}$ & $\begin{array}{l}\text { Nurse staffing factors: } \\
\text { float nurse: a nurse } \\
\text { normally assigned } \\
\text { elsewhere in the hospital } \\
\text { or from an outside } \\
\text { agency, among others } \\
\text { Median N/P ratio: } \\
\text { number of registered } \\
\text { nurses for each patient } \\
\text { Median patient care } \\
\text { assistant to patient ratio: } \\
\text { number of patient care } \\
\text { assistants per shift per } \\
\text { 100 patients }\end{array}$ & 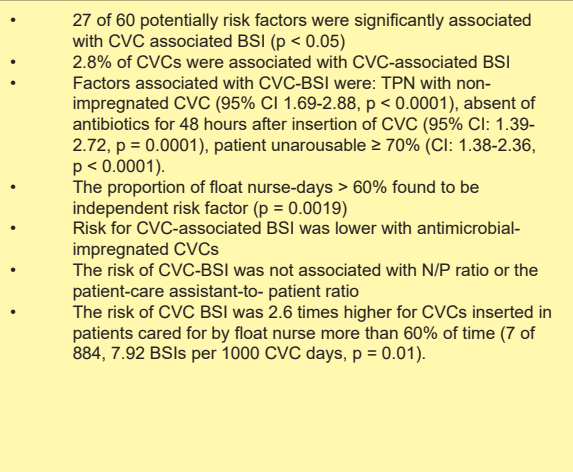 \\
\hline $\begin{array}{l}\text { Whitman et al/ 2002, } \\
\text { USA }\end{array}$ & $\begin{array}{l}\text { To describe the rates of } \\
\text { selected nurse sensitive } \\
\text { patient outcomes and } \\
\text { to determine if there } \\
\text { are differences in rates } \\
\text { across units }\end{array}$ & $\begin{array}{l}\text { Secondary } \\
\text { analyses of monthly } \\
\text { prospective } \\
\text { observational data } \\
95 \text { patient care units } \\
\text { across } 10 \text { adult } \\
\text { acute care hospitals } \\
\text { Data collection } \\
\text { methods were } \\
\text { different for each } \\
\text { NSO (monthly } \\
\text { surveillance data } \\
\text { from infection } \\
\text { control staff, monthly } \\
\text { system wide } 1 \\
\text { day prevalence, } \\
\text { pharmacy and } \\
\text { risk management } \\
\text { reports, patient' } \\
\text { interviews one day } \\
\text { every month, finance } \\
\text { office reports)/ } \\
1 \text { year study }\end{array}$ & $\begin{array}{ll}\text { - } & \text { CLBSI: number of CLIs/ } \\
\text { number of central line } \\
\text { days in place } \\
\text { PU: number of hospital } \\
\text { acquired PUs (grade } \\
\text { II or greater)/number of } \\
\text { patients assessed for } \\
\text { skin break down } \\
\text { Medication errors: } \\
\text { number of reported } \\
\text { medication errors/ } \\
\text { number of dispensed } \\
\text { doses } \\
\text { Falls: number of } \\
\text { unplanned descents } \\
\text { to the floorl/ number of } \\
\text { patient days } \\
\text { Patient satisfaction } \\
\text { with pain management: } \\
\text { percentage of patients } \\
\text { responding very } \\
\text { satisfied } \\
\text { Restraint application: } \\
\text { number of hours in } \\
\text { restraints/ number of } \\
\text { total hours available to } \\
\text { restrain patients }\end{array}$ & $\begin{array}{l}\text { Describes and } \\
\text { compares the rates } \\
\text { of already recognized } \\
\text { nurse sensitive patient } \\
\text { outcomes by ANA }\end{array}$ & 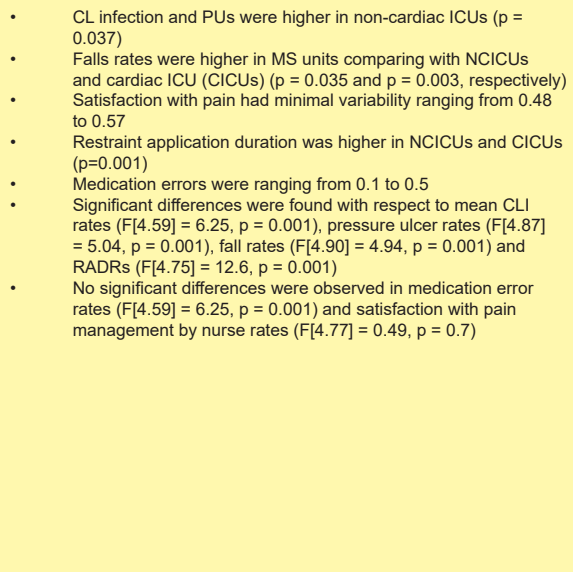 \\
\hline
\end{tabular}

Table 1. Continued 


\begin{tabular}{|c|c|c|c|c|c|}
\hline $\begin{array}{l}\text { Authors, } \\
\text { publication date, } \\
\text { country }\end{array}$ & Research aim(s) & $\begin{array}{l}\text { Methods: study } \\
\text { design, population, } \\
\text { tools used study } \\
\text { duration }\end{array}$ & $\begin{array}{l}\text { QIs and formula or } \\
\text { definition identified }\end{array}$ & $\begin{array}{l}\text { Nurse staffing variables } \\
\text { and definition identified }\end{array}$ & Results \\
\hline $\begin{array}{l}\text { Bracco et al., 2001, } \\
\text { Switzerland }\end{array}$ & $\begin{array}{l}\text { To determine the } \\
\text { occurrence of critical } \\
\text { incidents focusing on } \\
\text { incidents due to human } \\
\text { factors. Secondary aim } \\
\text { was to identify patients or } \\
\text { situations at risk and try } \\
\text { to determine clinical and } \\
\text { financial consequences } \\
\text { of human related } \\
\text { incidents }\end{array}$ & $\begin{array}{ll} & \text { Prospective } \\
\text { observational study } \\
\text { 1024 consecutive } \\
\text { patients admitted } \\
\text { in a 11-bed } \\
\text { multidisciplinary ICU } \\
\text { in a non- university } \\
\text { teaching 280- bed } \\
\text { hospital } \\
\text { A list including 105 } \\
\text { items (defining } \\
\text { critical incidents) } \\
\text { and a standardized } \\
\text { data sheet were } \\
\text { used to collect } \\
\text { data during clinical } \\
\text { round. Incidents } \\
\text { were analyzed within } \\
\text { 24hrs } \\
\text { 1 year study }\end{array}$ & $\begin{array}{ll}\text { Mortality } \\
\text { Readmissions } \\
\text { Critical incidence } \\
\text { Cost } \\
\text { LOS } \\
\text { Human errors: } \\
\text { venous lines and } \\
\text { catheters, respiratory } \\
\text { system, cardiovascular } \\
\text { system, drug related } \\
\text { complications, } \\
\text { neurological system } \\
\text { complications, urinary } \\
\text { system complications, } \\
\text { gastrointestinal } \\
\text { system complications, } \\
\text { skin and muscular } \\
\text { system, management } \\
\text { complications } \\
\text { All definitions of } \\
\text { critical incidents } \\
\text { and categorization } \\
\text { of complications } \\
\text { were listed including } \\
\text { diagnostic criteria }\end{array}$ & $\begin{array}{ll} & \text { Human errors } \\
\text { Regular nursing staff } \\
\text { amounted to } 3.2 \text { nurses } \\
\text { per bed. } \\
\text { Nursing staff included: } \\
\text { mix skilled, ICU certified } \\
\text { nurses, certified nurses } \\
\text { undergoing ICU training } \\
\text { and RNs without ICU } \\
\text { certification }\end{array}$ & 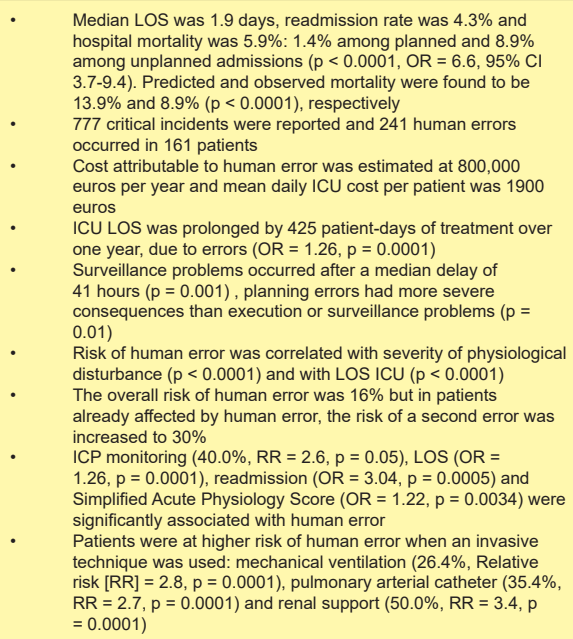 \\
\hline $\begin{array}{l}\text { Valentin et al., 2006, } \\
\text { world-wide }\end{array}$ & $\begin{array}{l}\text { To assess the prevalence } \\
\text { and corresponding } \\
\text { factors of selected } \\
\text { unintended events that } \\
\text { comprise patient safety } \\
\text { in ICU }\end{array}$ & $\begin{array}{l}\text { Observational, } \\
\text { prospective, } \\
\text { multinational study } \\
\text { of incidents. Cross- } \\
\text { sectional design } \\
1913 \text { patients adult } \\
\text { >18 years old in } \\
205 / 280 \text { ICUs from } \\
29 \text { countries and } 4 \\
\text { continents } \\
\text { Data were obtained } \\
\text { by: a structured } \\
\text { questionnaire } \\
\text { was used for } \\
\text { anonymous report of } \\
\text { unintended events; } \\
\text { a questionnaire } \\
\text { for information for } \\
\text { ICU characteristics, } \\
\text { patient and nurses' } \\
\text { related factors; and } \\
\text { the nine equivalents } \\
\text { of nursing manpower } \\
\text { use score (NEMS) } \\
\text { for nursing workload; } \\
\text { patient data which } \\
\text { were recorded using } \\
\text { a project website and } \\
\text { online data collection } \\
\text { software } \\
24 h r \text { observation } \\
\text { period }\end{array}$ & $\begin{array}{l}\text { Sentinel events. All } \\
\text { sentinel events were } \\
\text { presented as rates per } \\
100 \text { patient days } \\
\text { Medication errors: } \\
\text { - prescription; } \\
\text { administration; wrong } \\
\text { dose, drug, route } \\
\text { Airway: unplanned } \\
\text { extubation - artificial } \\
\text { airway obstruction; cuff } \\
\text { leakage; prompting } \\
\text { reintubation } \\
\text { Indwelling lines: iv } \\
\text { cannulas and the } \\
\text { attachment fluid } \\
\text { delivery sets; catheters: } \\
\text { arterial line, CVP, } \\
\text { pulmonary artery } \\
\text { catheters, folley, } \\
\text { dialysis; probes and } \\
\text { drains: unplanned } \\
\text { dislodgment, } \\
\text { inappropriate, } \\
\text { disconnection of chest } \\
\text { drain and nasogastric } \\
\text { tubes } \\
\text { Equipment failure: } \\
\text { infusion devices; } \\
\text { ventilator and } \\
\text { accessories; renal } \\
\text { replacement devices; } \\
\text { power and oxygen } \\
\text { supply } \\
\text { Alarms: inappropriate } \\
\text { turn off }\end{array}$ & $\begin{array}{ll}\text { - } & \text { Nursing workload as } \\
\text { calculated by NEMS } \\
\text { N/P ratio each shift }\end{array}$ & 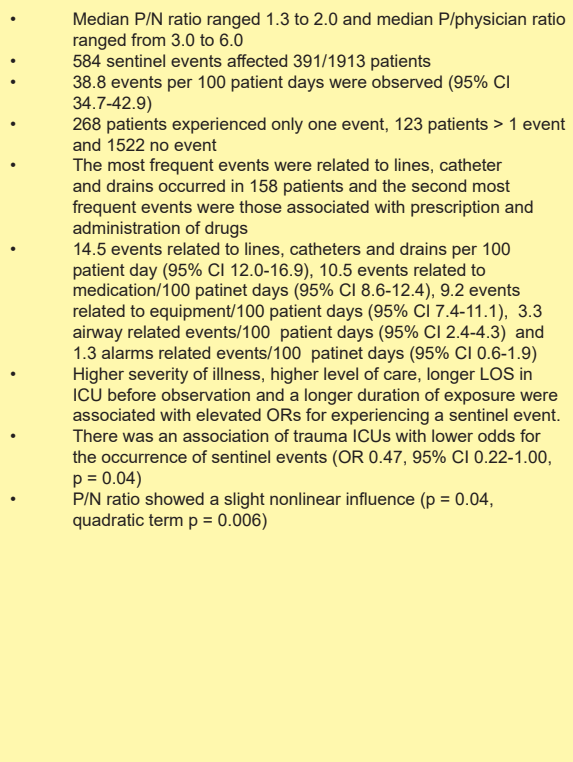 \\
\hline $\begin{array}{l}\text { Tarnow-Mordi et al., } \\
2000 \text {, Scotland }\end{array}$ & $\begin{array}{l}\text { To investigate whether } \\
\text { mortality is independently } \\
\text { related to nursing } \\
\text { requirement and other } \\
\text { measures of workload }\end{array}$ & $\begin{array}{ll}\text { - } & \text { Retrospective } \\
\text { analysis from a } \\
\text { prospective cohort } \\
\text { study } \\
1050 \text { adult episodes } \\
\text { representing } \\
1025 \text { patients (25 } \\
\text { readmissions) in } \\
1 \text { ICU } \\
\quad \text { Locally agreed } \\
\text { formula was used to } \\
\text { calculate the number } \\
\text { of appropriated } \\
\text { staffed beds } \\
\text { Nursing requirement } \\
\text { per shift was } \\
\text { recorded by senior } \\
\text { nurse at the end of } \\
\text { each shift according } \\
\text { to recommendations } \\
\text { of UK intensive care } \\
\text { society } \\
4 \text { year study }\end{array}$ & $\begin{array}{ll} & \text { Mortality: death in the } \\
\text { ICU or before discharge } \\
\text { from hospital } \\
\text { LOS } \\
\text { Readmission rates in } \\
\text { the ICU }\end{array}$ & 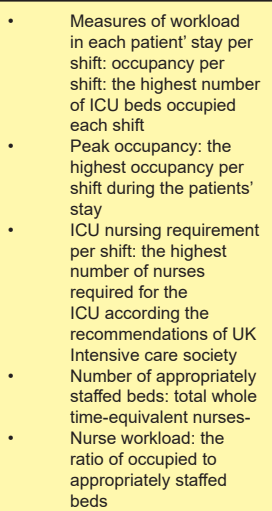 & $\begin{array}{l}337 \text { deaths were recorded (226 ICU, } 111 \text { before hospital } \\
\text { discharge) and } 61 \text { readmissions in a total of } 1286 \text { admissions } \\
\text { Median LOS was } 2.2 \text { days (0.3-95.8) } \\
\text { Median ratio of occupied beds to appropriately staffed beds } \\
\text { was } 1.3(0.4-2.2) \\
\text { Median nursing requirement per shift was } 9.2(2.5-14.9) \text {. } \\
\text { Median nursing requirement per occupied bed per shift was } \\
\text { 1.6 (0.7-2.3) } \\
\text { Adjusted mortality was related to the ratio of occupied of } \\
\text { appropriately staffed beds per shift, peak occupancy and ICU } \\
\text { nursing requirement per occupied bed per shift } \\
\text { Unadjusted mortality was greater for patients exposed to high vs } \\
\text { moderate overall ICU workload (OR = 4.0, 95\% CI 2.6-6.2) } \\
\text { Adjusted mortality was more than } 2 \text { times higher in patients } \\
\text { exposed to high workload (average nursing requirement per } \\
\text { occupied bed and peak occupancy) than those exposed to low } \\
\text { workload (OR = 3.1, } 95 \% \text { Cl 1.9-5.0) }\end{array}$ \\
\hline
\end{tabular}

Table 1. Continued 


\begin{tabular}{|c|c|c|c|c|c|}
\hline $\begin{array}{l}\text { Authors, } \\
\text { publication date, } \\
\text { country }\end{array}$ & Research aim(s) & $\begin{array}{l}\text { Methods: study } \\
\text { design, population, } \\
\text { tools used study } \\
\text { duration }\end{array}$ & $\begin{array}{l}\text { Qls and formula or } \\
\text { definition identified }\end{array}$ & $\begin{array}{l}\text { Nurse staffing variables } \\
\text { and definition identified }\end{array}$ & Results \\
\hline $\begin{array}{l}\text { Garcia \& Fugulin, } \\
\text { 2012, Brazil; USA }\end{array}$ & $\begin{array}{l}\text { To analyze the time } \\
\text { the nursing team uses } \\
\text { to see patients in ICU, } \\
\text { as well as to check its } \\
\text { correlation with quality } \\
\text { care indicators }\end{array}$ & 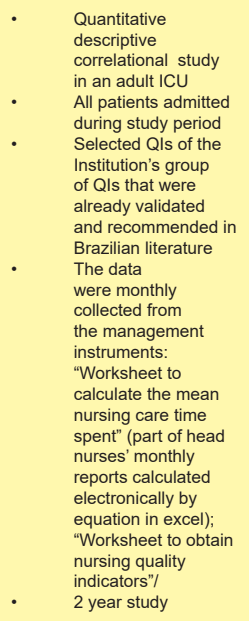 & 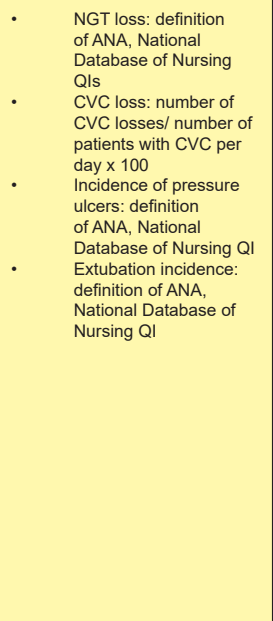 & $\begin{array}{l}\text { Nursing time (including } \\
\text { nurses and nursing } \\
\text { technicians) spent to } \\
\text { assist each patient: } \\
\text { mean number of nursing } \\
\text { staff members in each } \\
\text { professional category } \\
\text { X mean productivity } \\
\text { of each professional } \\
\text { category } X \text { work journey } \\
\text { of each profossional } \\
\text { category }+ \text { mean daily } \\
\text { number of patients } \\
\text { attended }\end{array}$ & 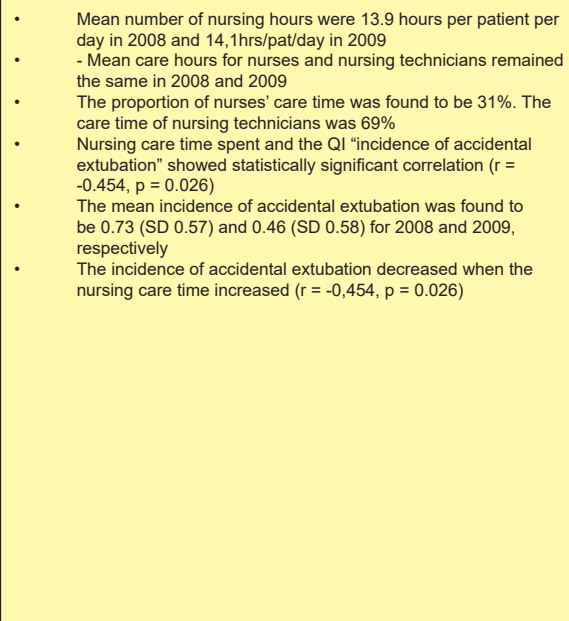 \\
\hline $\begin{array}{l}\text { Metnitz et al., 2008, } \\
\text { Austria }\end{array}$ & $\begin{array}{l}\text { To evaluate the } \\
\text { relationship between } \\
\text { patient volume and } \\
\text { outcome (survival status } \\
\text { at ICU and hospital } \\
\text { discharge status) in a } \\
\text { large cohort of critically } \\
\text { ill patients }\end{array}$ & $\begin{array}{ll}\text { - } & \text { Prospective, } \\
\text { multicenter cohort } \\
\text { study } \\
\text { 83259 patients from } \\
\text { 40 ICUs in Austria } \\
\text { A questionnaire was } \\
\text { used to examine } \\
\text { the structural } \\
\text { characteristics } \\
\text { of quality of the } \\
\text { included ICUs } \\
\text { ASDI prospectively } \\
\text { collected data } \\
7 \text { year study }\end{array}$ & $\begin{array}{ll}\text { Fatal outcome } \\
\text { Observed to expected } \\
\text { mortality: number of } \\
\text { observed deaths per } \\
\text { group/ the number } \\
\text { of SAPS II predicted } \\
\text { deaths per group } \\
\text { ICU LOS } \\
\text { ICOspital LOS }\end{array}$ & $\begin{array}{l}\text { - Level of the provided } \\
\text { care (assessed by } \\
\text { TISS } 28 \text { ) } \\
\text { P/N ratio: number of } \\
\text { patients assigned to } \\
\text { one nurse } \\
\text { The eficient use of } \\
\text { nursing personnel was } \\
\text { evaluated from the work } \\
\text { utilization ratio and was } \\
\text { calculated by specific } \\
\text { formula } \\
\text { Occupancy rate: the } \\
\text { percentage of occupied } \\
\text { beds per day }\end{array}$ & 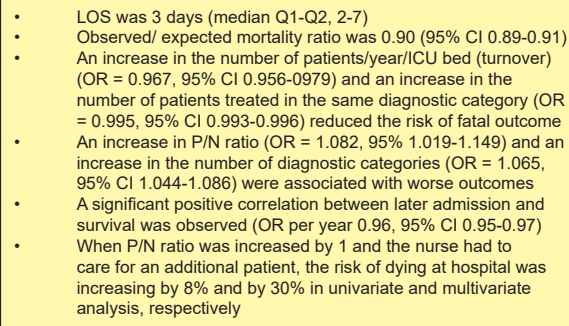 \\
\hline $\begin{array}{l}\text { Stone et al., 2007, } \\
\text { USA }\end{array}$ & $\begin{array}{l}\text { To examine effects of a } \\
\text { comprehensive set of } \\
\text { working conditions on } \\
\text { elderly patient safety } \\
\text { outcomes in ICU }\end{array}$ & 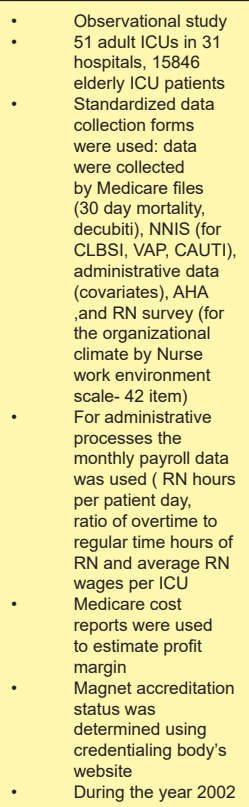 & 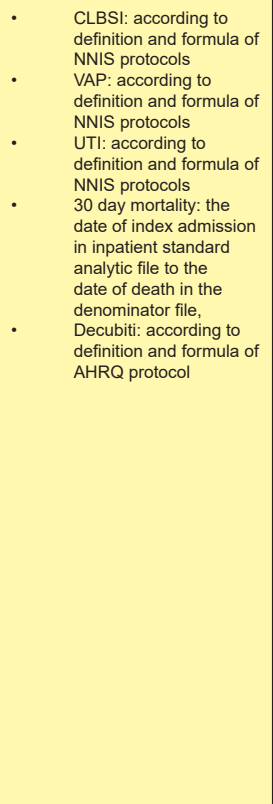 & $\begin{array}{ll}\cdot & \text { RN hours per patient } \\
\text { day } & \text { day patio of overtime to } \\
\text { ref } & \text { regular time hrs of RNs } \\
\text { - Average RN wages } \\
\text { per ICU } \\
\text { Average RN wages } \\
\text { per ICU } \\
\text { Overtime } \\
\text { Effective work conditions } \\
\text { (by the organizational } \\
\text { climate calculated by } \\
\text { Nursing work scale } \\
\text { (NWS) }\end{array}$ & 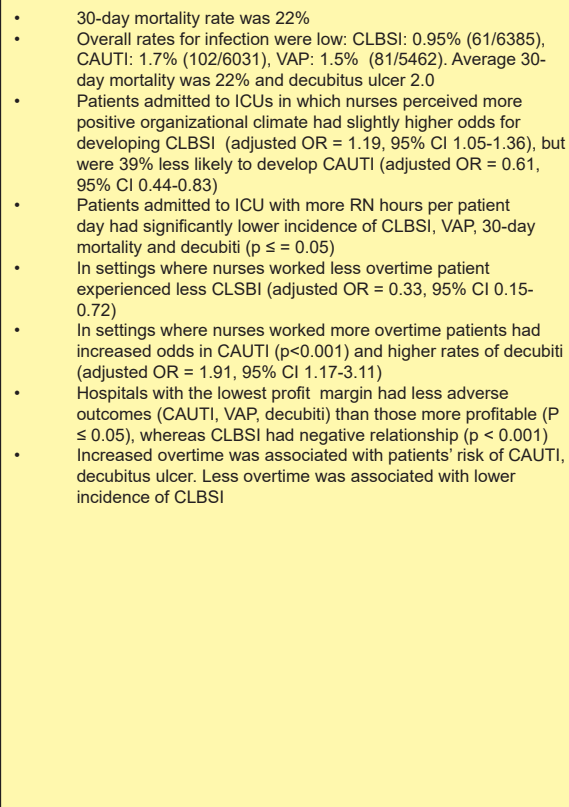 \\
\hline
\end{tabular}

Table 1. Continued. $\mathrm{AHA}=$ American Hospital Association (annual survey data), ANA = American Nurses Association, ASDI = Austrian Center for Documentation and Quality Assurance, BSI = bloodstream infection, CAUTI = catheter-associated urinary tract infection, $\mathrm{CDC}=$ Centers of Disease Control and Prevention, $\mathrm{Cl}=\mathrm{Confidence} \mathrm{interval,} \mathrm{CIMC}=$ cardiac intermediate care units, CCLI $=$ central catheter line infection, CLBSI = central line bloodstream infections, ICD= International Center of Disease, ICU = intensive care unit, ICP = intracranial pressure , IMC = intermediate care unit, LOS = length of stay, MAE= medication administrator error, MS = medical/surgical, NCICU = Non-cardiac ICU, NEMS = Nine Equivalents of Nursing Manpower Use Score, NNIS = National Nosocomial Infections Surveillance, NNPR $=$ night nurse to patient ratio, N/P ratio $=$ nurse to patient ratio, NWS $=$ Nursing Work Scale, NNIS $=$ nosocomial infections surveillance, OR $=$ odds ratio, $p=$ significance value, $\mathrm{RADR}=$ restraint application duration rate, $\mathrm{RN}=$ registered nurse, SAPS $=$ Simplified Acute Physiology Score, UE $=$ unplanned extubation, $\mathrm{UTI}=\mathrm{urinary}$ tract infections, $\mathrm{Yrs}=\mathrm{Years}, \mathrm{Vs}=\mathrm{Versus}$ 


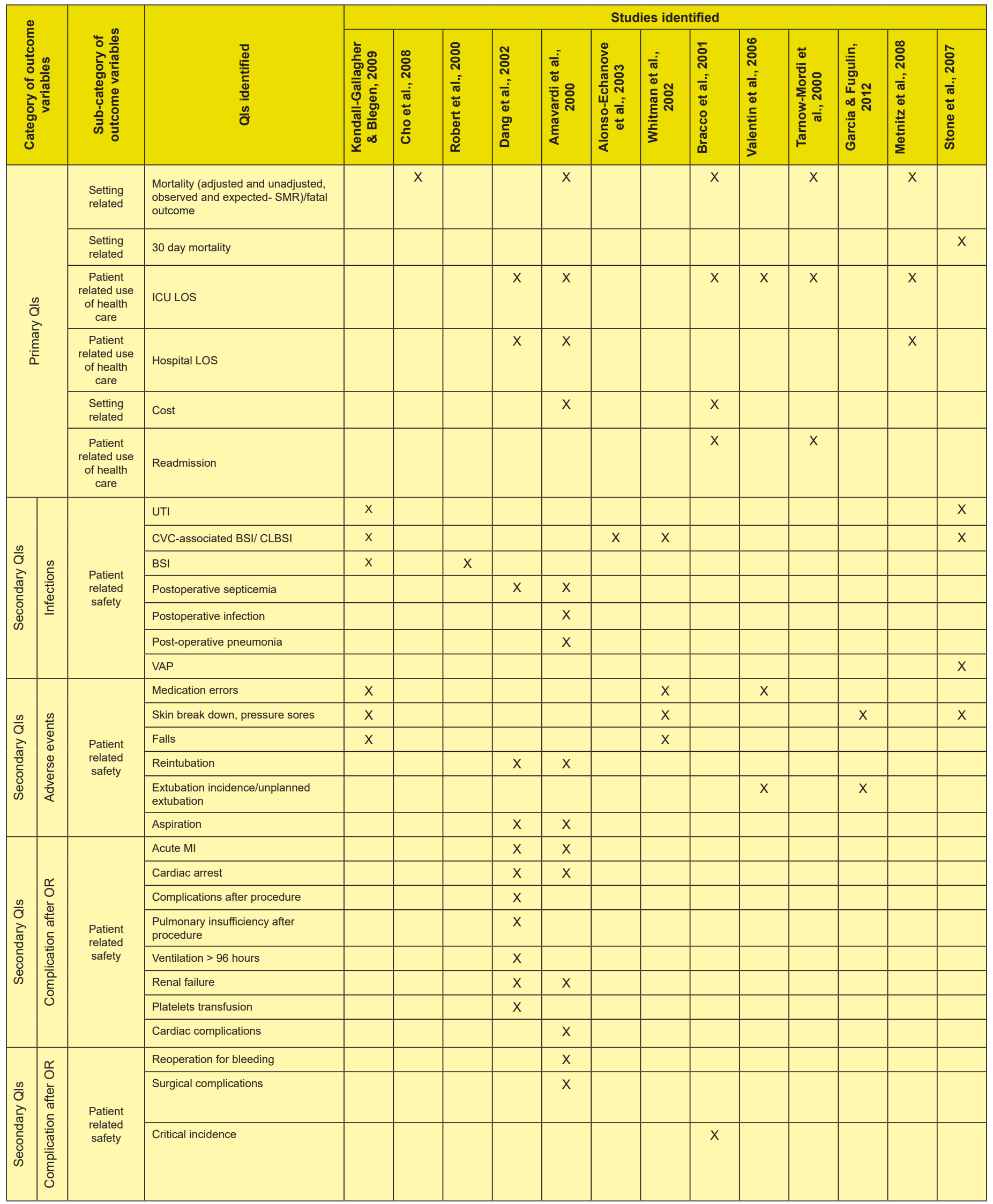

Table 2. Categorization of quality indicators identified in the included studies 


\begin{tabular}{|c|c|c|c|c|c|c|c|c|c|c|c|c|c|c|c|c|}
\hline \multirow{2}{*}{\multicolumn{2}{|c|}{ 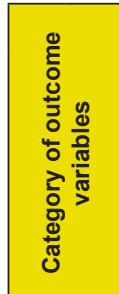 }} & \multirow[b]{2}{*}{ 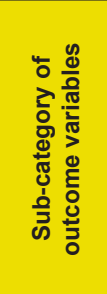 } & \multirow[b]{2}{*}{ 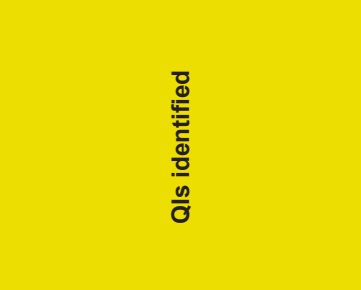 } & \multicolumn{13}{|c|}{ Studies identified } \\
\hline & & & & 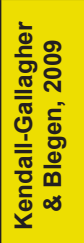 & 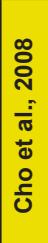 & 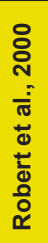 & 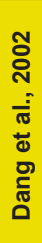 & 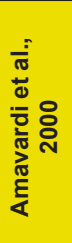 & 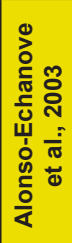 & 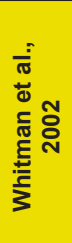 & 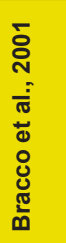 & 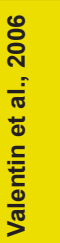 & 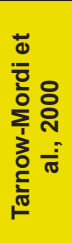 & 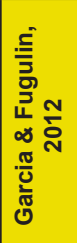 & 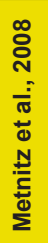 & 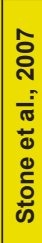 \\
\hline \multirow{9}{*}{$\begin{array}{l}\frac{\infty}{0} \\
⿱ 亠 乂 \\
\frac{\pi}{0} \\
0 \\
\delta \\
\delta \\
\infty \\
\infty\end{array}$} & \multirow{9}{*}{ 商 } & \multirow{9}{*}{$\begin{array}{l}\text { Patient } \\
\text { related } \\
\text { safety }\end{array}$} & $\begin{array}{l}\text { Human errors related to venous lines } \\
\text { and catheters }\end{array}$ & & & & & & & & $x$ & & & & & \\
\hline & & & $\begin{array}{l}\text { Human errors related to respiratory } \\
\text { system }\end{array}$ & & & & & & & & $x$ & & & & & \\
\hline & & & $\begin{array}{l}\text { Human errors related to } \\
\text { cardiovascular system }\end{array}$ & & & & & & & & $x$ & & & & & \\
\hline & & & $\begin{array}{l}\text { Human errors related to drug related } \\
\text { complications }\end{array}$ & & & & & & & & $x$ & & & & & \\
\hline & & & $\begin{array}{l}\text { Human errors related to neurological } \\
\text { system complications }\end{array}$ & & & & & & & & $x$ & & & & & \\
\hline & & & $\begin{array}{l}\text { Human errors related to urinary } \\
\text { system complications }\end{array}$ & & & & & & & & $x$ & & & & & \\
\hline & & & $\begin{array}{l}\text { Human errors related to } \\
\text { Gastrointestinal system } \\
\text { complications }\end{array}$ & & & & & & & & $x$ & & & & & \\
\hline & & & $\begin{array}{l}\text { Human errors related to skin and } \\
\text { muscular system }\end{array}$ & & & & & & & & $x$ & & & & & \\
\hline & & & $\begin{array}{l}\text { Human errors related to } \\
\text { management complications }\end{array}$ & & & & & & & & $x$ & & & & & \\
\hline \multirow{2}{*}{\multicolumn{2}{|c|}{$\begin{array}{l}\frac{\pi}{0} \\
\frac{\pi}{0} \\
\overline{0} \frac{\omega}{0} \\
0 \\
\infty\end{array}$}} & $\begin{array}{l}\text { Patient } \\
\text { related } \\
\text { perception }\end{array}$ & $\begin{array}{l}\text { Patient satisfaction with pain } \\
\text { management }\end{array}$ & & & & & & & $x$ & & & & & & \\
\hline & & $\begin{array}{l}\text { Patient } \\
\text { related } \\
\text { safety }\end{array}$ & Restraint application & & & & & & & $\mathrm{x}$ & & & & & & \\
\hline \multirow{4}{*}{$\begin{array}{l}\frac{\infty}{0} \\
\geq \\
\frac{\pi}{\pi} \\
0 \\
\overline{0} \\
\mathbb{d} \\
\infty\end{array}$} & \multirow{4}{*}{ 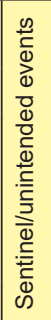 } & \multirow{4}{*}{$\begin{array}{l}\text { Patient } \\
\text { related } \\
\text { safety }\end{array}$} & $\begin{array}{l}\text { Sentinel events related to medication } \\
\text { (prescription, administration) }\end{array}$ & & & & & & & & & $x$ & & & & \\
\hline & & & Sentinel events related to airway & & & & & & & & & $x$ & & & & \\
\hline & & & $\begin{array}{l}\text { Sentinel events related to indwelling } \\
\text { lines (lines, catheters, drains, arterial } \\
\text { lines CVP lines, pulmonary artery } \\
\text { catheters, folley catheters, dialysis } \\
\text { catheters, chest drain, nasogastric } \\
\text { tubes) }\end{array}$ & & & & & & & & & $x$ & & $x$ & & \\
\hline & & & Sentinel events related to equipment & & & & & & & & & $\mathrm{x}$ & & & & \\
\hline
\end{tabular}

Table 2. Continued

\section{DISCUSSION}

The current review builds on previous research pooling patients' outcomes associated with the quality and quantity of nursing care in the ICU. This review examines the methodological quality of QIs identified, through a relative new instrument (AIRE) (De Roo et al., 2013). To the authors' knowledge, this is the first time that the AIRE instrument is used for the methodological quality assessment of NQIs in the ICU.

The QIs identified concerned a specific health care setting (ICU), even though they were previously used for other health care settings as well. The heterogeneity was evident both with regards to the number of QIs, which ranged from 1 to 15 , as well as to the type of QIs measured (Tables 1 and 2). The observed variation across QIs indicates that the selection of Qls depends to some extent on the type and needs of each ICU, as well as the purpose the QIs are selected to serve. The composition of involved stakeholders and the defined criteria may also affect the selection of Qls. The geographical origin, the main causes of mortality of the population under study and the available means need to be taken into consideration (Mainz, 2003). Additionally, critically ill patients' outcomes are not equally sensitive to nursing care (Whitman et al., 2002). The examination of a specific group in the ICU, as well as the diagnostic related group are factors that may affect the sensitivity to nursing care and the selection of QIs. Qls that describe the positive effect of nursing care delivered by measures of improved health status, such as symptom control (e.g. dyspnea, nausea) were not identified. This finding may suggest that this type of information is not recorded and documented in administrative databases (Savitz et al., 2005). Only Whitman et al. (2002) reported rates of "patient satisfaction with pain management". Likewise, there seems to be a lack of patient- reported outcome QIs, which should be addressed in future studies.

Methodological characteristics of the identified QIs varied considerably across the included studies (Table 3). The high scores obtained in the category "scientific evidence" were indicative of the fact that generally valid and widely used QIs were included in these studies. On the other hand, low scores in the remaining categories suggest that future studies should pay more attention to reporting the involvement of relevant stakeholders as well as to providing full descriptions of the Qls used.

Structure variables associated with patient outcomes differ among included studies (Table 1). This was also highlighted in previously published reviews (Numata et al., 2006; Penoyer, 2010; West et al., 


\begin{tabular}{|c|c|c|c|c|c|c|c|c|c|c|c|c|c|c|c|c|c|}
\hline \multirow[b]{2}{*}{ Items } & \multirow[b]{2}{*}{ Dimension } & \multicolumn{13}{|c|}{ Studies identified } & \multirow[b]{2}{*}{ 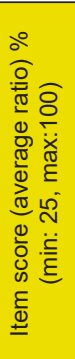 } & \multirow[b]{2}{*}{ 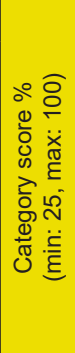 } & \multirow[b]{2}{*}{ 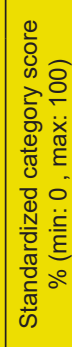 } \\
\hline & & 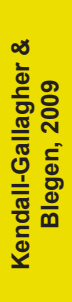 & 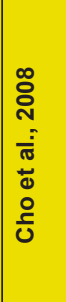 & 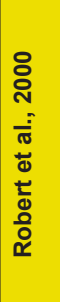 & 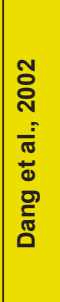 & 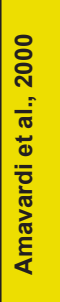 & 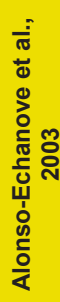 & 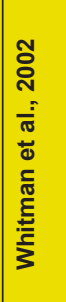 & 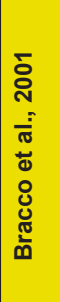 & 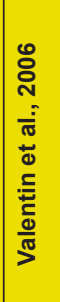 & 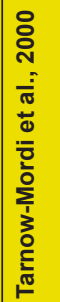 & 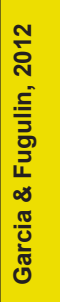 & 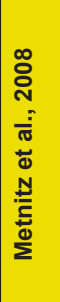 & 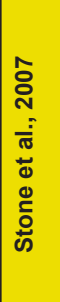 & & & \\
\hline \multirow{5}{*}{ 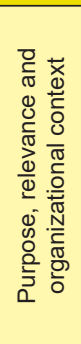 } & $\begin{array}{l}\text { The purpose of the indicator is described } \\
\text { clearly }\end{array}$ & 11 & 15 & 16 & 12 & 12 & 15 & 15 & 14 & 14 & 15 & 16 & 14 & 11 & 87 & \multirow{5}{*}{81} & \multirow{5}{*}{75} \\
\hline & $\begin{array}{l}\text { The criteria for selecting the topic of the } \\
\text { indicator are described in detail }\end{array}$ & 10 & 11 & 13 & 11 & 14 & 14 & 15 & 13 & 13 & 14 & 14 & 13 & 12 & 80 & & \\
\hline & $\begin{array}{l}\text { The organizational context of the indicator } \\
\text { is described in detail }\end{array}$ & 8 & 9 & 10 & 11 & 9 & 10 & 9 & 12 & 13 & 9 & 10 & 11 & 12 & 64 & & \\
\hline & $\begin{array}{l}\text { The quality domain the indicator } \\
\text { addresses is described in detail }\end{array}$ & 13 & 6 & 16 & 16 & 15 & 15 & 12 & 15 & 15 & 15 & 12 & 11 & 14 & 83 & & \\
\hline & $\begin{array}{l}\text { The health care process covered by the } \\
\text { indicator is described and defined in detail }\end{array}$ & 14 & 13 & 15 & 14 & 14 & 15 & 15 & 15 & 15 & 15 & 15 & 16 & 16 & 92 & & \\
\hline \multirow{3}{*}{ 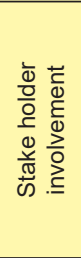 } & $\begin{array}{l}\text { The group developing the indicator } \\
\text { includes individuals from all relevant } \\
\text { professional groups }\end{array}$ & 4 & 4 & 4 & 9 & 10 & 4 & 4 & 4 & 4 & 6 & 4 & 4 & 4 & 31 & \multirow{3}{*}{51} & \multirow{3}{*}{35} \\
\hline & $\begin{array}{l}\text { Considering the purpose of the } \\
\text { indicator, all relevant stakeholders have } \\
\text { been involved at some stage of the } \\
\text { development process }\end{array}$ & 4 & 4 & 4 & 16 & 9 & 8 & 4 & 4 & 4 & 5 & 4 & 4 & 4 & 36 & & \\
\hline & The indicator has been formally endorsed & 16 & 4 & 16 & 16 & 15 & 14 & 4 & 15 & 15 & 13 & 16 & 12 & 16 & 88 & & \\
\hline \multirow{3}{*}{ 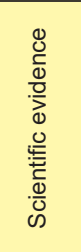 } & $\begin{array}{l}\text { Systematic methods were used to search } \\
\text { for scientific evidence }\end{array}$ & 11 & 11 & 14 & 12 & 13 & 15 & 13 & 13 & 13 & 14 & 14 & 13 & 16 & 83 & \multirow{3}{*}{89} & \multirow{3}{*}{85} \\
\hline & $\begin{array}{l}\text { The indicator is based on } \\
\text { recommendations from an evidence } \\
\text { based guideline or studies published in } \\
\text { peer-reviewed scientific journals }\end{array}$ & 16 & 16 & 16 & 11 & 16 & 16 & 15 & 15 & 15 & 16 & 16 & 15 & 16 & 96 & & \\
\hline & $\begin{array}{l}\text { The supporting evidence has been } \\
\text { critically appraised }\end{array}$ & 16 & 16 & 15 & 11 & 14 & 14 & 15 & 14 & 14 & 13 & 14 & 14 & 15 & 89 & & \\
\hline \multirow{9}{*}{ 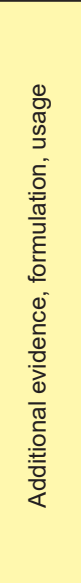 } & $\begin{array}{l}\text { The numerator and denominator are } \\
\text { described in detail }\end{array}$ & 6 & 15 & 16 & 10 & 10 & 15 & 14 & 10 & 10 & 14 & 13 & 13 & 12 & 75 & \multirow{9}{*}{86} & \multirow{9}{*}{2} \\
\hline & $\begin{array}{l}\text { The target patient population of the } \\
\text { indicator is defined clearly }\end{array}$ & 11 & 15 & 16 & 15 & 16 & 16 & 11 & 12 & 12 & 13 & 12 & 14 & 16 & 86 & & \\
\hline & $\begin{array}{l}\text { A strategy for risk adjustment has been } \\
\text { considered and described }\end{array}$ & 8 & 16 & 14 & 16 & 14 & 14 & 10 & 13 & 13 & 13 & 10 & 13 & 15 & 81 & & \\
\hline & $\begin{array}{l}\text { The indicator measures what it is } \\
\text { intended to measure (validity) }\end{array}$ & 14 & 14 & 16 & 12 & 14 & 16 & 12 & 14 & 14 & 16 & 14 & 12 & 16 & 88 & & \\
\hline & $\begin{array}{l}\text { The indicator measures accurately and } \\
\text { consistently (reliability) }\end{array}$ & 14 & 14 & 15 & 12 & 14 & 16 & 15 & 14 & 14 & 16 & 14 & 11 & 16 & 89 & & \\
\hline & $\begin{array}{l}\text { The indicator has sufficient discriminative } \\
\text { power }\end{array}$ & 14 & 12 & 14 & 15 & 11 & 14 & 13 & 12 & 12 & 13 & 11 & 14 & 15 & 82 & & \\
\hline & The indicator has been piloted in practice & 16 & 16 & 16 & 16 & 16 & 16 & 16 & 14 & 14 & 16 & 14 & 15 & 16 & 97 & & \\
\hline & $\begin{array}{l}\text { The efforts needed for data collection } \\
\text { have been considered }\end{array}$ & 14 & 15 & 15 & 16 & 15 & 15 & 16 & 14 & 14 & 14 & 14 & 14 & 15 & 92 & & \\
\hline & $\begin{array}{l}\text { Specific instructions for presenting and } \\
\text { interpreting results }\end{array}$ & 11 & 16 & 15 & 12 & 14 & 15 & 14 & 14 & 14 & 14 & 13 & 13 & 15 & 87 & & \\
\hline
\end{tabular}

Table 3. Quality assessment of the quality indicators identified through research and evaluation (AIRE) instrument (De Roo et al., 2013). Category score $=$ (sum of individual authors' scores for the items in a category) / (maximum possible score for that category); Standardized category score (range $0-100 \%)=$ (total score per category - minimum possible score per category) / (maximum possible score per category - minimum possible score per category)

2009). Almost half of the studies examined N/P ratio. Nevertheless, it should be noted that, even when the same nurse staffing variable was considered, it was measured in different ways, for example N/P ratio in the morning versus night shift (Amavardi et al., 2000; Dang et al., 2002). It is evident that there are many different ways of measuring nurses' contribution to patient care. However, this variability makes comparison among the studies difficult. It is of note that in more than half of the studies, it is not clear if nurses are registered nurses (RNs) or if other levels of nursing personnel were included.

A wide variability regarding mortality and morbidity rates was also observed (Table 1). This variation may be related to differences regarding organizational and structural factors of the ICUs, including available resources, capacity, type of each ICU, as well as care processes and policies, for instance admission and discharge criteria (Pronovost et al., 2003). Furthermore, it is of note that there are no universally applied systems that enable constant collection of QIs. Similarly, there are no uniform definitions and descriptions of QIs (Whitman et al., 2002). Thus, similar QIs may lead to different calculations because the formulas as well as inclusion and exclusion criteria provided by different organizations may vary (AHRQ 2006). 
The findings of this review suggest that the increased ratio of average daily census to the total number of full time equivalent RNs (ADC/RNs) and N/P ratio at day and night, overtime, workload, use of float or pool nurses and the low intensity staffing are all strongly associated with higher rates of adverse events, mortality, infections and complications in adult ICUs (Table 1). These findings are consistent with previous results in acute and critical care populations (Griffiths et al., 2008, Numata et al., 2006, Penoyer, 2010, Van den Heede et al., 2007, West et al., 2009).

Sets that assess ICU performance have been previously described (Najjar-Pellet et al., 2008). Furthermore, there are sets of Qls that focus on specific populations of the ICU: neurological (Russell et al., 2002), end-stage (Clarke et al., 2003), palliative care (Mularski et al., 2006), medical and surgical (Pronovost et al., 2003) and cardiology patients (Idemoto and Kresevic, 2007). The current literature review did not reveal a complete set of QIs according to structure-processoutcome, which can quantify nursing care in the ICU. However, the NQIs identified are evidence-based and valid. Thus, they could be used in the context of the development of a unique set of NQIs specific to the ICU. To achieve this end-goal, a well-defined multilevel and structured research approach is needed that combines the available evidence with expert opinion.

\section{Limitations}

The use of MESH terms probably limited retrieval of results (Pronovost et al., 1999). In particular, using the general term QI as a key word probably restricted the articles yielded. Furthermore, the review did not employ any method for retrieving grey literature. Even though a number of additional studies from European countries (Isfort, 2013) and Korea (Choi et al., 2008) were identified in the search, those were not published in the English language, and were thus excluded. To the authors' knowledge, the AIRE instrument has not been used previously in the context of assessing QIs in the ICU. Thus, no comparisons could be drawn. Lastly, the inclusion of articles published after 2000 may have limited the number of articles examined; however, the review has covered a relatively long time period.

\section{CONCLUSIONS}

ICU patients may be at greater risk when the overall nursing staff performance is disturbed (West et al., 2009). Mishaps indicate poor quality of the care provided. Continuous assessment of measures, which quantify the level of nursing quality and nursing contribution to patients' care outcomes is pivotal. The NQIs pooled in this review could be used for the development of a unique set specific to the ICU. Monitoring of validated NQIs may improve critically ill patients' outcomes that are mostly affected by nurses. This would also allow the comparison between ICU settings. A set of NQIs specific for the ICU may influence clinical nursing practice, guide improvements of the care delivered and contribute to the transformation and improvement of the health care system (De Vos. et al., 2007, Numata et al., 2006, Penoyer, 2010). Lastly, monitoring of NQIs in a unit recognizes that the critically ill patient remains the focus of the care process.

\section{REFERENCES}

Agency of Health Care Research and Quality (2006). AHRQ Quality indicators. Guide to patient safety indicators, Version 3. [Online] Available at: http://www.qualityindicators.ahrq.gov Accessed 5 May 2007.

Alonso-Echanove J, Edwards JR, Richards MJ, et al. (2003). Effect of nurse staffing and antimicrobial-impregnated central venous catheters on the risk for bloodstream infections in intensive care units. Infection Control and Hospital Epidemiology 24(12), 916-925.

Amaravadi RK, Dimick JB, Pronovost PJ, Lipsett PA (2000). ICU nurseto-patient ratio is associated with complications and resource use after esophagectomy. Intensive Care Medicine 26(12), 1857-1862.
American Nurses Association (2000). Nurse staffing and patient outcomes in the inpatient hospital setting. American Nurses Publishing: Washington.

Bracco D, Favre JB, Bissonnette B, et al. (2001). Human errors in a multidisciplinary intensive care unit: a 1-year prospective study. Intensive Care Medicine 27 (1), 137-145.

Braun JP, Hendrik M, Hanswerner B et al. (quality network in intensive care medicine) (2010). Quality indicators in intensive care medicine: why? Use or burden for the intensivist. GMS German Medical Science 8(22), 1-20.

Brook RH, McGlynn EA, Cleary PD (1996). Quality of health care. part 2: measuring quality of care. New England Journal of Medicine 335 (13), 966-70.

Center for Reviews and Dissemination (2009). Systematic reviews. CRD's guidance for undertaking reviews in health care. CRD, University of York: York. pp. 6-90.

Cho SH, Hwang JH, Kim J (2008). Nurse staffing and patient mortality in intensive care units. Nursing research 57(5), 322-330.

Choi YJ, Lim JY, Lee YW, Kim HS (2008). Development of nursing key performance indicators for an intensive care unit by using a balanced score card. Journal of Korean Academic Nursing 38(5), 656-66.

Clarke EB, Curtis JR, Luce JM, Levy M, Danis M, Nelson J, Solomon MZ (2003). Quality indicators for end-of-life care in the intensive care unit. Critical Care Medicine 31 (9), 2255-62.

Dang D, Johantgen ME, Pronovost PJ, Jenckes MW, Bass EB (2002). Postoperative complications: does intensive care unit staff nursing make a difference? Heart and Lung 31(3), 219-228.

De Bruin-Kooistra M, Amelink-Verburg MP, Buitendijk SE, Westert GP (2012). Finding the right indicators for assessing quality midwifery care. Intnational Journal of Quality in Health Care 24(3), 301-310.

De Roo ML, Leemans K, Claessen SJ, et al. (2013). Quality indicators for palliative care: update of systematic review. Journal of Pain and Symptom Management 46(4), 556-572.

De Vos M, Graafmans W, Keesman E, Westert G, Van der Voort PH (2007). Quality measurement at intensive care units: which indicators should we use? Journal of Critical Care 22(4), 267- 274.

Delgado MCM, Pericas LC, Moreno JR, et al. (2005). Quality indicators in critically ill patients. Madrid: SEMICYUC.

Donabedian A (1992). The role of outcomes in quality assessment and assurance. Quality Review Bulletin 18(11), 356-60.

Doran DM (2003). "Preface", In: Doran DM (editor), Nursing-Sensitive Outcomes: State of the Science. MA: Jones and Bartlett, Sudbury, pp. vii-ix.

Doran DM, Pringle D (2011). Patient outcomes as accountability, In: Doran DM (editor), Nursing Outcomes: The State of the Science, 2nd edition. MA: Jones and Bartlett Learning, Sudbury, pp. 11-20.

Garcia PC, Fugulin FMT (2012). Nursing care time and quality indicators for adult intensive care: correlation analysis. Revista Latino-americana de Enfermagem 20(4), 651-658.

Griffiths P, Jones S, Maben J, Murrells T (2008). State of the art metrics for nursing: a rapid appraisal. National Nursing Research Unit. King's College London: University of London, pp. 2-16. [Online] Available at: http://www. $\mathrm{kcl}$.ac.uk/schools/nursing/nnru.

Heslop L, Lu S (2014). Nursing-sensitive indicators: a concept analysis. Journal of Advanced Nursing 70(11), 2469-2482.

Holzemer WL (1994). The impact of nursing care in Latin America and the Caribbean: a focus on outcomes. Journal of Advanced Nursing 20(1), 5-12.

Idemoto B, Kresevic D (2007). Emerging Nurse-Sensitive Outcomes and Evidence-Based Practice in Postoperative Cardiac Patients. Critical Care Nursing Clinics of North America 19(4), 371-384.

Isfort M (2013). Influence of personnel staffing on patient care and nursing in German ICUs. Descriptive study on aspects of patient safety and stress indicators of nursing. [German]. Medizinischa Klinik, Intensivmedizin und Notfallmedizin 108(1), 71-77.

Joint Commission (2007). Performance measurement initiatives. The JointCommission. [Online] Available at: www.jointcommission.org/ PerformanceMeasurement/PerformanceMeasurement/ Accessed 27 May 2007. 
Kendall-Gallagher D, Blegen MA (2009). Competence and certification of registered nurses and safety of patients in intensive care units. American Journal of Critical Care 18(2), 106-114.

Mainz J (2003). Developing evidence-based clinical indicators: a state of the art methods primer. International Journal for Quality in Health Care 15(suppl. 1), 5-11.

McGahan M, Kucharski G, Coyer F (2012). Nurse staffing levels and the incidence of mortality and morbidity in the adult intensive care unit: a literature review. Australian Critical Care 25(2), 64-77.

Metnitz B, Metnitz PGH, Bauer P, Valentin A (2008). Patient volume affects outcome in critically ill patients. Wiener Klinische Wochenschrift. The Middle European Journal of Medicine 121(1-2), 34-40.

Mitchell PH (2008). Defining patient safety and quality care, In: Hughes, R.G. (ed), Patient safety and quality: An evidence-based handbook for nurses. AHRQ (US): Rockville.

Montalvo I (2007). The national database of nursing quality indicators (NDNQI). Home ANA Periodicals, OJIN: the on online journal of issues in nursing 12(3).

Mularski RA, Curtis JR, Billings JA, et al. (2006). Proposed quality measures for palliative care in the critically ill: A consensus from the Robert Wood Johnson Foundation. Critical Care Workgroup. Critical Care Medicine 34(11), 404-411.

Najjar-Pellet J, Jonquet O, Jambou P, Fabry J (2008). Quality assessment in intensive care units: proposal for a scoring system in terms of structure and process. Intensive Care Medicine 34(2), 278-85.

National Quality Forum (2004). National Voluntary Consensus Standards for Nursing-Sensitive Care: An Initial Performance measure set. A consensus report by the National Quality Forum. NQF, Washington: USA, pp. 1-14.

Needleman J, Buerhaus P, Mattke S, Stewart M, Zelevinsky K (2002). Nurse-staffing levels and the quality of care in hospitals. The New England Journal of Medicine 346(22), 1715-1722.

Numata Y, Schulzer M, Van der Wal R., et al. (2006). Nurse staffing levels and hospital mortality in critical care settings: literature review and metaanalysis. Journal of Advanced Nursing 55(4), 435-48.

Penoyer DA (2010). Nurse staffing and patient outcomes in critical care: A concise review. Critical Care Medicine 38(7), 1521-1528.

Petrosyan Y, Sahakyan Y, Barnsley J, Kuluski K, Liu B, Wodchis W (2017). Quality indicators for care of osteoarthritis in primary care settings: a systematic literature review. Family Practice 35(2), 151-159.

Pronovost PJ, Berenholtz SM, Ngo K, et al. (2003). Developing and pilot testing quality indicators in the intensive care unit. Journal of Critical Care 18(3), 145-155.

Pronovost PJ, Jenckes MW, Dorman T, et al. (1999). Organizational characteristics of intensive care units related to outcomes of abdominal aortic surgery. Journal of the American Medical Association 281(14), 13101317.

Ray B, Samaddar DP, Todi SK, Ramakrishnan N, John G, Ramasubban S (2009). Quality indicators for ICU: ISCCM guidelines for ICUs in India. Indian Journal of Critical Care Medicine 13(4), 173-206. [Online] Available at: http://www.ijccm.org/text.asp?2009/13/4/173/60172
Robert J, Fridkin SK, Blumberg HM, et al. (2000). The influence of the composition of the nursing staff on primary bloodstream infection rates in a surgical intensive care unit. Infection Control and Hospital Epidemiology 21(1), 12-7.

Russell D, Vorder-Bruegge M, Burns S (2002). Effect of an outcomes managed approach to care of neuroscience patients by acute care nurse practitioners. American Journal of Critical Care 11(4), 353-362.

Savitz LA, Jones CB, Bernard S (2005). Quality indicators sensitive to nurse staffing in acute care settings, In: Henriksen K, Battles JB, Marks ES, Lewin DI (Eds), Advances in patient safety: from research to implementation, Volume 4: programs, tools and products. Rockville: Agency for Healthcare Research and Quality (US), pp. 375-385.

Smeulers M, Verweij L, Maaskant JM, et al. (2015) Quality indicators for safe medication preparation and administration: a systematic review. PLOS One 10(4), 1-14. [Online] Available at: https://doi.org/10.1371/journal. pone.0122695

Stone PW, Mooney-Kane C, Larson EL, et al. (2007). Nurse working conditions and patient safety outcomes. Medical Care 45(6), 571-578.

Strudwick K, Nelson M, Martin-Khan M, Bourke M, A Bell, Russell T (2015). Quality indicators for musculoskeletal injury management in the emergency department: a systematic review. Academic Emergency Medicine 22(2), 127-141.

Tarnow-Mordi WO, Hau C, Warden A, Shearer J (2000). Hospital mortality in relation to staff workload: a 4 year study in an adult ICU. The Lancet 356(9225), 185-189.

Valentin A, Capuzzo M, Guidet B, et al. (2006). Patient safety in intensive care: results from the multinational sentinel events evaluation (SEE) study. Intensive Care Medicine 32(10), 1591- 1598.

Van den Heede K, Clarke SP, Sermeus W, Vleugels A, Aiken LH (2007). International experts' perspectives on the state of the nurse staffing and patient outcomes literature. Journal of nursing scholarship 39(4), 290-297.

West E, Mays N, Rafferty AM, Rowan K, Sanderson C (2009). Nursing resources and patient outcomes in intensive care: a systematic review of the literature. International Journal of Nursing Studies 46(7), 993-1011.

West S, King V, Carey TS, et al. (2002). Systems to rate the Strength of scientific evidence, in: Prepared by the Research Triangle InstituteUniversity of North Carolina Evidence-based Practice Center under Contract No. 290-97-0011. Evidence Report/Technology Assessment No. 47 AHRQ. Publication No. 02-E016. Rockville: Agency for Healthcare Research and Quality, pp. 17-68.

Whittemore R, Knafl K (2005). The integrative review: updated methodology. Journal of Advanced Nursing 52(5), 546-553.

Whitman GB, Kim Y, Davidson LJ, Wolf GA, Wang SL (2002). Measuring nurse- sensitive patient outcomes across specialty units. Outcomes Management 6(4), 152-158. 